\title{
Using proteomic profiling to characterize protein signatures of different thymoma subtypes
}

\author{
Liang-Chuan Lai ${ }^{1,3+}{ }^{+}$Qiang-Ling Sun ${ }^{2 \dagger}$, Yu-An Chen ${ }^{3}$, Yi-Wen Hsiao ${ }^{3}$, Tzu-Pin Lu ${ }^{4}$, Mong-Hsun Tsai ${ }^{3,5}$, Lei Zhu ${ }^{7}$,
} Eric Y. Chuang ${ }^{3,6,8^{*}}$ (D) and Wentao Fang ${ }^{2^{*}}$

\begin{abstract}
Background: Histology is a traditional way to classify subtypes of thymoma, because of low cost and convenience. Yet, due to the diverse morphology of thymoma, this method increases the complexity of histopathologic classification, and requires experienced experts to perform correct diagnosis. Therefore, in this study, we developed an alternative method by identifying protein biomarkers in order to assist clinical practitioners to make right classification of thymoma subtypes.

Methods: In total, 204 differentially expressed proteins in three subtypes of thymoma, AB, B2, and B3, were identified using mass spectrometry. Pathway analysis showed that the differentially expressed proteins in the three subtypes were involved in activation-related, signaling transduction-related and complement system-related pathways. To predict the subtypes of thymoma using the identified protein signatures, a support vector machine algorithm was used. Leave-oneout cross validation methods and receiver operating characteristic analysis were used to evaluate the predictive performance.

Results: The mean accuracy rates were $>80 \%$ and areas under the curve were $\geqq 0.93$ across these three subtypes. Especially, subtype B3 had the highest accuracy rate (96\%) and subtype AB had the greatest area under the curve (0.99). One of the differentially expressed proteins COL17A2 was further validated using immunohistochemistry.

Conclusions: In summary, we identified specific protein signatures for accurately classifying subtypes of thymoma, which could facilitate accurate diagnosis of thymoma patients.
\end{abstract}

Keywords: Proteomic profiling, Thymoma, Support vector machine, WHO classification

\section{Background}

Epithelial tumors of the thymus include thymoma and thymic carcinoma. Compared to thymic carcinoma, thymoma tends to recur locally and is frequently associated with autoimmune diseases, such as myasthenia gravis. The incidence rate of thymoma is around 2.5 per million people per year [1]. The age distribution ranges from 10 to 80 years old with an average age of 50 to 60 in both males and females [1].

\footnotetext{
* Correspondence: chuangey@ntu.edu.tw; vwtfang12@shchest.org †Liang-Chuan Lai and Qiang-Ling Sun contributed equally to this work. ${ }^{3}$ Bioinformatics and Biostatistics Core, Center of Genomic and Precision Medicine, National Taiwan University, Taipei 10055, Taiwan

${ }^{2}$ Department of Thoracic Surgery, Shanghai Chest Hospital, Shanghai Jiao Tong University, Shanghai 200030, China

Full list of author information is available at the end of the article
}

In the quest for accurate diagnosis, treatment, and prognosis for thymoma patients, researchers use clinical data to classify thymoma into different types. In 1999, the World Health Organization (WHO) proposed a classification system that divided thymic tumors into six types according to the morphology of epithelial cells and the lymphocyte-to epithelial cell ratio: thymoma type A, $\mathrm{AB}, \mathrm{B} 1, \mathrm{~B} 2$, and type $\mathrm{C}$ [2]. Type $\mathrm{C}$ thymoma was then revised into thymic carcinoma instead in the 2004 version of WHO thymoma classification standard [3, 4].

In this system, the existence of spindled or epithelioid neoplastic epithelial cells and the amount of lymphocytes was used as a basic classifying criterion. Also, Suster et al. proposed a thymoma classification system based on the differentiation level of tumor cells [5]. In this classification system, thymoma, atypical thymoma, and thymic

(c) The Author(s). 2019 Open Access This article is distributed under the terms of the Creative Commons Attribution 4.0 International License (http://creativecommons.org/licenses/by/4.0/), which permits unrestricted use, distribution, and 
carcinoma are defined as highly, moderately, and poorly differentiated, respectively. However, distinguishing thymic tumors merely through histological observations is still quite complicated because of the diverse morphology of thymoma and the presence of non-neoplastic lymphocytes. For example, type AB, B2 and B3 are all admixed with various amount of lymphocytes, but the prognosis of type $A B$ was different from those of type $B 2$ and $B 3$ [6]. Furthermore, due to the rarity of the disease, lack of experience among pathologists in general were quite common. Therefore, Suster et al. has reviewed several classification systems of thymic epithelial neoplasms, including Suster \& Moran, WHO, Kirchner \& MullerHermelink, and Bernatz, and suggested that the future of thymoma classification should include a simplified nomenclature rather than increasing complexity of histopathologic classification [2, 5, 7-9].

With the development of sequencing techniques in the twenty-first century, the Cancer Genome Atlas (TCGA) also provide thymoma sequencing data for genomic exploration [10, 11]; and the Clinical Lung Cancer Genome Project [12] as a genetic foundation for more accurate classification [13]. Also, Zettl et al. performed comparative genomic hybridization in 28 thymoma and 9 thymic squamous cell carcinoma cases. They found distinct genetic phenotypes between thymoma type A and type B3 [14]. Lee et al. also performed comparative genomic hybridization in 39 thymoma cases, and identified a set of 33 genes that could be divided into 4 genetically distinct groups $(\mathrm{A}, \mathrm{AB}, \mathrm{B} 1+2$, and $\mathrm{B} 3)$ according to the WHO classification, where type $A B$ was determined to be genetically heterogeneous [15]. These reports indicate the importance of and trend toward integrating clinical data with genomics and proteomics data for a more comprehensive understanding of thymoma classification.

Therefore, the purpose of this study was to classify three thymoma types (AB, B2, and B3), which were difficult to classify histologically, by analyzing the proteomics data of 21 Chinese patients. In total, 204 differentially expressed proteins were detected in the three subtypes of thymoma, and their functions were identified. Also, a predictive model for thymoma subtype was created, and leave-oneout cross validation (LOOCV) methods and receiver operating characteristic (ROC) curve analysis were used to evaluate the performance of the predictive model. Lastly, immunohistochemistry was used for validation.

\section{Methods}

\section{Collection and processing of clinical material and patient information}

Fresh tumor and adjacent normal tissue specimens were obtained during surgery between 1994 and 2010 and snap-frozen. The specimens were obtained from 21 patients treated at the Shanghai Chest Hospital, Shanghai Jiao Tong University, Shanghai, China. Tumor histology was determined according to the WHO classification [16] by an experienced pathologist (LZ) who reviews over a hundred cases of thymic malignancies annually. Information regarding age, gender, pathological stage, and WHO classification was collected prospectively. The study was approved by the Institutional Review Board of Shanghai Chest Hospital. And written consent was acquired from all patients included in this study.

\section{Mass spectrometry analysis}

In contrast to shotgun mass spectrometry (MS) and traditional database searches, the quantitative proteomic profiling of type $\mathrm{AB}$, type $\mathrm{B} 2$ and type $\mathrm{B} 3$ tissues were performed by using Data Independent Acquisition Mass Spectrometry (DIA-MS) to expand the detectable dynamic range and improve the overall confidence of protein quantification measurements. Fresh frozen tissues were lysed and subjected to reduction, alkylation, and tryptic digestion. Peptides were then subjected to desalt using C18 SPE 96-well plates prior to LCMS/MS analysis using Orbitrap Fusion (Thermo Scientific). One $\mu$ g peptides was loaded onto a nano-C18 column and separated at a flow rate of $300 \mathrm{~nL} / \mathrm{min}$. Data independent high-resolution MS/MS spectra were acquired by sequential $25 \mathrm{amu}$ window. Protein identification and quantification were processed by Spectronaut software (Biognosys Inc.). Differential expressed proteins were subjected to bioinformatics analysis.

\section{Data analysis}

Raw protein expression intensities were preprocessed by $\log _{2}$ transformation and then normalized by quantile normalization. To remove the background noise, proteins whose normalized intensity was below 32 were removed from further analysis, which accounted for $\sim 2.5 \%$ of the total protein in each sample. Then, differentially expressed proteins (DEPs) between tumor and adjacent normal tissue in each subtype were identified by $\log _{2}$ fold-change value $\geqq 7$ and $P$-value $<0.05$ using Wilcoxon signed-rank test. After selecting DEPs from each subtype, the total and unique (subtype-specific) DEPs were identified and visualized by Venn diagrams. Principal component analysis using the expression values of total DEPs was used to visualize the similarity of different samples. An unsupervised hierarchical clustering method was applied using Euclidean distance and the method of average linkage in Genesis version 1.8.1 (http://genome.tugraz.at/genesisclient/genesisclient_description.shtml). Ingenuity Pathway Analysis (IPA) was used to identify enriched pathways of DEPs for each subtype.

To predict the subtypes of thymoma by identified protein signatures, a support vector machine (SVM) 
algorithm, a supervised learning model, was used. The unique DEPs in each subtype were chosen as a set of features for training the classifiers. In this study, our classifiers are the three thymoma subtypes, the closer to real results in the prediction, the more accurate classifiers they are. During the LOOVC analysis, each set of unique DEPs of one subtype took turns serving as testing data, whereas the remaining two sets served as training data. After the predictive models were established (three models in total), all three subtypes were then served as three sets of sample data, yielding nine predictive results. The robustness of each classifier was estimated by the average classification accuracy rate in each validation. After LOOCV, the sensitivity, specificity, positive predictive value, and negative predictive value of the prediction models for each subtype were calculated. The evaluation of these parameters was performed using ROC curve analysis, the result of which was quantified by computing the area under the curve (AUC).

\section{Immunohistochemical analysis}

Five type $A B$ and five type $B 2 / B 3$ thymoma formalinfixed and paraffin-embedded (FFPE) tissue sections were used to examine the presence of COL17A2. Briefly, slides were deparaffinized with diaminobenzene at $65^{\circ} \mathrm{C}$ for $2 \mathrm{~h}$, and rehydrated in graded alcohol solutions. The tissue sections were then treated with $3 \%$ hydrogen peroxidase to block endogenous peroxidase activity. After washing in PBS solution, the tissue sections were incubated in $20 \%$ normal goat serum at $37^{\circ} \mathrm{C}$ for $10 \mathrm{~min}$. Rabbit monoclonal antihuman COL17A2 antibody (Abcam ab184996) was applied at 1:100 dilution overnight. Biotin- and streptavidin-labeled antibodies were used for 3,3'-diaminobenzidine staining. Nucleus was further stained with hematoxylin. Images were taken by ZEISS fluorescent microscope.

The score system was based on the immunoreactive intensity to COL17A2 antibody and the percentage of positively stained cells. The stained intensity was scored from a value from 0 to 3 . The percentage of positively stained cells were the average score in 5 fields (100 cells per field in 400x magnitude). Score 4 was given for $>$ $75 \%$ of positive stained cells; score 3 for $75-51 \%$ of cells positive; score 2 for $50-26 \%$ of cells positive; score 1 for $25-6 \%$ of cells positive; score 0 when less, than $5 \%$ of tumor cells or no visible staining was observed. The immunoreactive score is determined by multiplication of the score of staining intensity with the score of percentage of positively stained cells.

\section{Results}

Patients

The clinicopathological characteristics of the 21 thymoma patients included in this study are summarized in
Table 1. The median of age was 51. Tumor stages and histology subtypes were defined by Masaoka-Koga system [17] and WHO classification separately. There was no significant difference in the distribution of patients regarding gender, stage, or subtype according to WHO classification.

\section{Identification of differentially expressed proteins}

In total, 204 DEPs between tumor and adjacent normal tissue of the 3 thymoma subtypes were identified through statistical filtering (Table 2). The number of DEPs detected in AB, B2, and B3 subtypes was 97, 103, and 114, respectively. The number of up-/down-regulated DEPs and unique (subtype-specific) DEPs is shown in Table 2. The distribution of the total number of DEPs among shared and unique DEPs is shown in Fig. 1a.

\section{Cluster analysis}

Principal component analysis of the 21 paired samples (tumor versus normal, 42 dots in total) was performed using a total of 204 DEPs in 3 subtypes (Fig. 1b). The normal and tumor samples were clearly divided into 2 main groups, and within the tumor cluster, the B2 and $B 3$ subtypes were further distinguished from the AB subtype, with the exception of one B3 sample appearing with the $A B$ cluster. This result suggests a distinct protein expression pattern in the $A B$ subtype of thymoma as compared to the other 2 subtypes.

Hierarchical cluster analysis was performed within each subtype-unique DEPs set (Fig. 1c). The expression level of each unique DEPs set (labeled on the right) in all three subtypes (labeled on the top) was presented by

Table 1 Clinicopathological characteristics of patients with thymoma. Tumor stages are defined by Masaoka-Koga classification

\begin{tabular}{ll}
\hline Factors & Number (\%) \\
\hline Age & \\
$\geqq 60$ & $6(28.6)$ \\
$<60$ & $15(71.4)$ \\
Gender & \\
Male & $10(47.6)$ \\
Female & $11(52.4)$ \\
Stage & \\
I & $9(42.9)$ \\
II & $7(33.3)$ \\
III & $2(9.5)$ \\
IV & $3(14.3)$ \\
WHO classification & \\
AB & $8(38.1)$ \\
B2 & $8(38.1)$ \\
B3 & $5(23.8)$ \\
\hline
\end{tabular}


Table 2 Number of differentially expressed proteins between thymoma and adjacent normal tissue

\begin{tabular}{llll}
\hline & \multicolumn{2}{l}{ Thymoma Type } & \\
\cline { 2 - 4 } & $\mathrm{AB}$ & $\mathrm{B} 2$ & $\mathrm{~B} 3$ \\
\hline All proteins & 3118 & 103 & 114 \\
DEP $($ T vs N) & 97 & 33 & 34 \\
Up-regulation & 40 & 70 & 80 \\
Down-regulation & 57 & 42 & 37 \\
Unique & 43 & & \\
\hline
\end{tabular}

${ }^{a}$ T: tumor tissue; $\mathrm{N}$ : adjacent normal tissue

${ }^{b}$ DEP: differentially expressed protein. Selection criteria: absolute $\log _{2}$ foldchange value $\geq 7$ and a $P$-value $\leq 0.05$ using a Wilcoxon signed-rank test heatmap. The expression difference of each unique DEPs set was apparently greater in their own subtype than in other 2 subtypes, confirming the existence of unique sets of DEPs for each subtype.

\section{Pathway analysis}

IPA revealed the biological pathways in which the DEPs participated (Table 3). A $-\log P$-value of Fisher's exact test and the ratio of DEPs to the total number of proteins in the same pathway were used to estimate the significance and importance of each pathway. Among the top 5 canonical pathways in each thymoma subtype (15 pathways total), the most frequently occurring were signal transduction (6 pathways), LXR/RXR or FXR/RXR activation (4 pathways), and complement system (2 pathways).

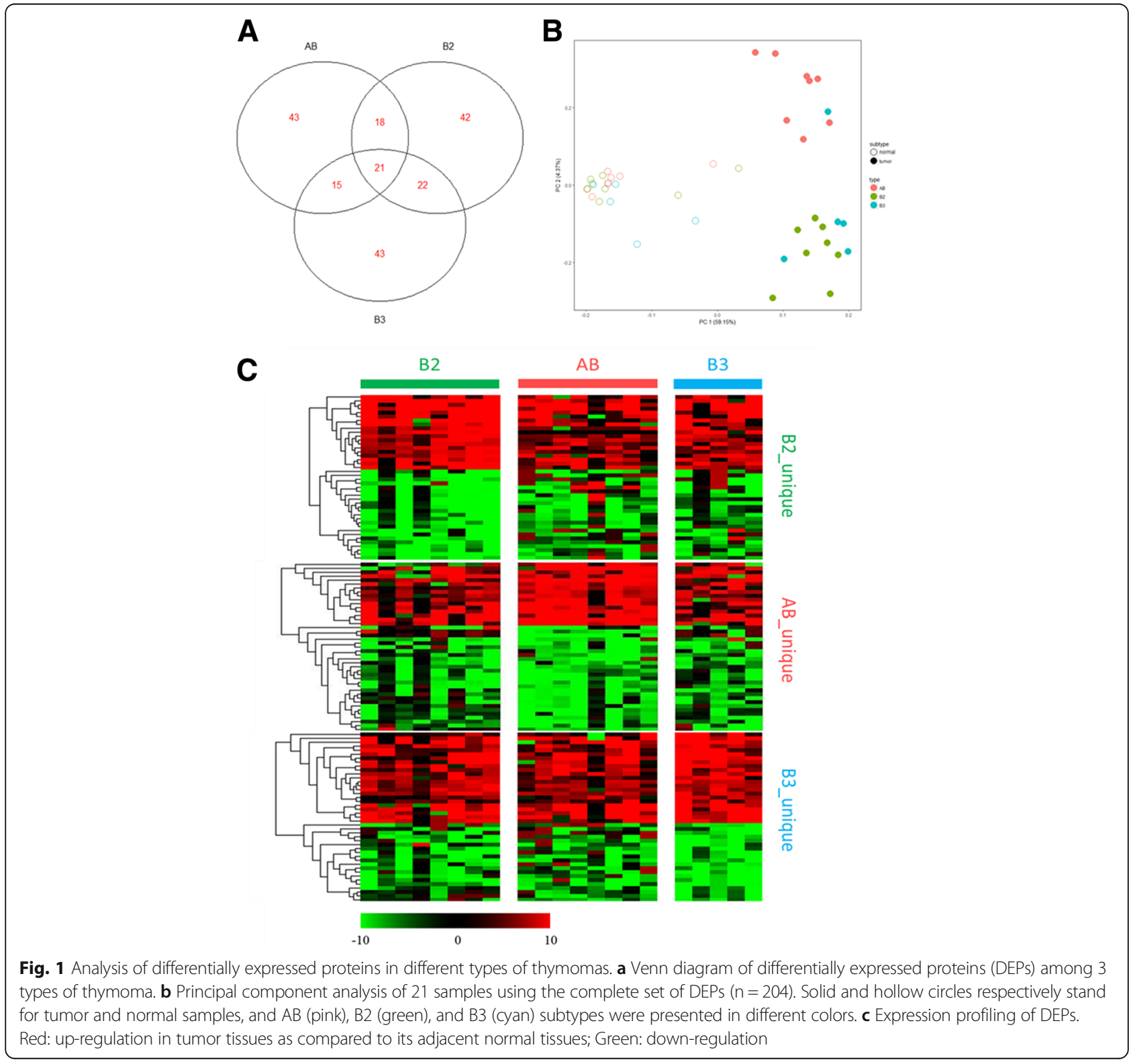


Table 3 Pathway analysis of differentially expressed proteins for each subtype of thymoma

\begin{tabular}{|c|c|c|}
\hline Canonical Pathway $^{a}$ & $\begin{array}{l}\text {-log } \\
(P \text {-value })^{b}\end{array}$ & Ratioc $^{c}$ \\
\hline \multicolumn{3}{|l|}{ Subtype $A B$} \\
\hline Acute Phase Response Signaling & 6.96 & 0.05 \\
\hline Complement System & 3.18 & 0.08 \\
\hline Apoptosis Signaling & 3.07 & 0.04 \\
\hline Triacylglycerol Degradation & 3.05 & 0.08 \\
\hline Granzyme B Signaling & 2.59 & 0.13 \\
\hline \multicolumn{3}{|l|}{ Subtype B2 } \\
\hline LXR/RXR Activation & 8.06 & 0.07 \\
\hline FXR/RXR Activation & 6.71 & 0.06 \\
\hline Atherosclerosis Signaling & 5.57 & 0.06 \\
\hline $\begin{array}{l}\text { Production of Nitric Oxide and Reactive Oxygen } \\
\text { Species in Macrophages }\end{array}$ & 4.31 & 0.04 \\
\hline IL-12 Signaling and Production in Macrophages & 4.10 & 0.04 \\
\hline \multicolumn{3}{|l|}{ Subtype B3 } \\
\hline Acute Phase Response Signaling & 12.00 & 0.08 \\
\hline Complement System & 7.83 & 0.17 \\
\hline Coagulation System & 4.69 & 0.11 \\
\hline LXR/RXR Activation & 4.68 & 0.05 \\
\hline FXR/RXR Activation & 4.60 & 0.05 \\
\hline
\end{tabular}

${ }^{a}$ Canonical pathway analysis was conducted by the Ingenuity ${ }^{\oplus}$ Pathway Analysis (IPA) program and analyzed based on the Ingenuity ${ }^{\oplus}$ Knowledge Base (Content version: 39480507; Release date: 2017-09-14)

${ }^{b}$ Fisher's exact test was used to determine the enrichment of differentially expressed proteins in a given canonical pathway

${ }^{c}$ Ratio represents the number of differentially expressed proteins in the pathway divided by the total number of proteins in the same pathway

When comparing common pathways between subtypes, subtypes B2 and B3 had 2 common pathways: LXR/RXR activation (- $\log P$-value $=8.06(\mathrm{~B} 2)$ and 4.68 (B3)) and FXR/RXR activation ( $-\log P$-value $=6.71$ (B2) and 4.60 (B3)). Subtypes AB and B3 also had 2 common pathways: acute phase response signaling $(-\log P$-value $=$ $6.96(\mathrm{AB})$ and $12.00(\mathrm{~B} 3))$ and complement system (-log $P$-value $=3.18(\mathrm{AB})$ and $7.83(\mathrm{~B} 3))$.

Subtype AB had the most similar pathways, where 3 of the top 5 canonical pathways were signaling-related: acute phase response signaling, apoptosis signaling, and granzyme B signaling $(-\log P$-value $=6.96,3.07$ and 2.59 ).

\section{Predicting thymoma subtypes with unique DEPs signatures}

The results of thymoma subtype prediction by a supervised learning model with existing unique protein signatures are shown in Table 4. The accuracy rate of LOOCV for all 3 subtypes was $>80 \%$, where B3 had the highest accuracy rate (96\%). Sensitivity, specificity, positive predictive value and negative predictive value are also shown (Table 4). The prediction efficiency
Table 4 Summary of thymoma subtype prediction by a LOOCV method

\begin{tabular}{|c|c|c|c|}
\hline & Thymo & & \\
\hline & $\overline{A B}$ & B2 & B3 \\
\hline No. of Samples & 8 & 8 & 5 \\
\hline No. of Unique DEP ${ }^{a}$ & 43 & 42 & 37 \\
\hline LOOCV mean accuracy rate ${ }^{b}$ & $83 \%$ & $83 \%$ & $96 \%$ \\
\hline Sensitivity & 0.89 & 0.73 & 0.50 \\
\hline Specificity & 1.00 & 1.00 & 0.86 \\
\hline PPV & $100 \%$ & $100 \%$ & $38 \%$ \\
\hline NPV ${ }^{d}$ & $97 \%$ & $91 \%$ & $91 \%$ \\
\hline$A_{U C}{ }^{e}$ & 0.99 & 0.93 & 0.94 \\
\hline
\end{tabular}

${ }^{a}$ DEP: differentially expressed protein

${ }^{b}$ The average classification accuracy rate was calculated in leave-one-out cross validation using differentially expressed proteins

c PPV: positive predictive value, the proportion of positive results in a classifier that is truly positive in the experimental results

${ }^{d} \mathrm{NPV}$ : negative predictive value, the proportion of negative results in a classifier that is truly negative in the experimental results

${ }^{e}$ AUC: Area under the curve, represents how well a classifier can distinguish one type from the others

evaluations of the diagnostic test by ROC analysis are shown in Fig. 2, with the AUCs of the SVM classifiers in Table 4. Overall, AB (AUC =0.99) seemed to be the best classifier among the 3 subtypes when comparing to other two subtypes, B2 (AUC = 0.93) and B3 in Fig. 2.

Lastly, we selected two of differentially expressed proteins COL17A1 (Collagen Type XVII Alpha 1 Chain) and TBR1 (T-Box, Brain 1) for validation using immunochemical analysis. Based on the results of mass spectrometry, COL17A1 was only up-regulated in type $A B$, but not in type $B 2 / B 3$, and had the greatest fold-change ( 920X). As shown in Fig. 3, COL17A1 was differentially expressed in subtype AB using immunohistochemistry (Fig. 3a). The immunoreactive score is determined by multiplication of the score of staining intensity with the score of percentage of positively stained cells. The amount and the percentage of thymoma containing COL17A1 was significantly $(P<0.01)$ higher in type $\mathrm{AB}$ as compared to type $\mathrm{B} 2 / \mathrm{B} 3$ (Fig. 3b). In addition, immunofluorescence of TBR1 validated the results of mass spectrometry (Fig. 3c), which showed TBR1 had significantly higher expression in type B2/B3 than type AB.

\section{Discussion}

Because of the diverse morphology of thymoma and the rarity of the disease, classification of thymoma solely based on histology is challenging. Here we used proteomics information to identify DEPs in different thymoma subtypes in order to classify them. This method can be used to customize the diagnosis, treatment, and prognosis of specific thymoma subtypes. In our results, distinct protein expression patterns of thymoma subtype $A B$ were revealed, as compared to B2 and B3 subtypes, in both cluster analysis and pathway analysis. Furthermore, 

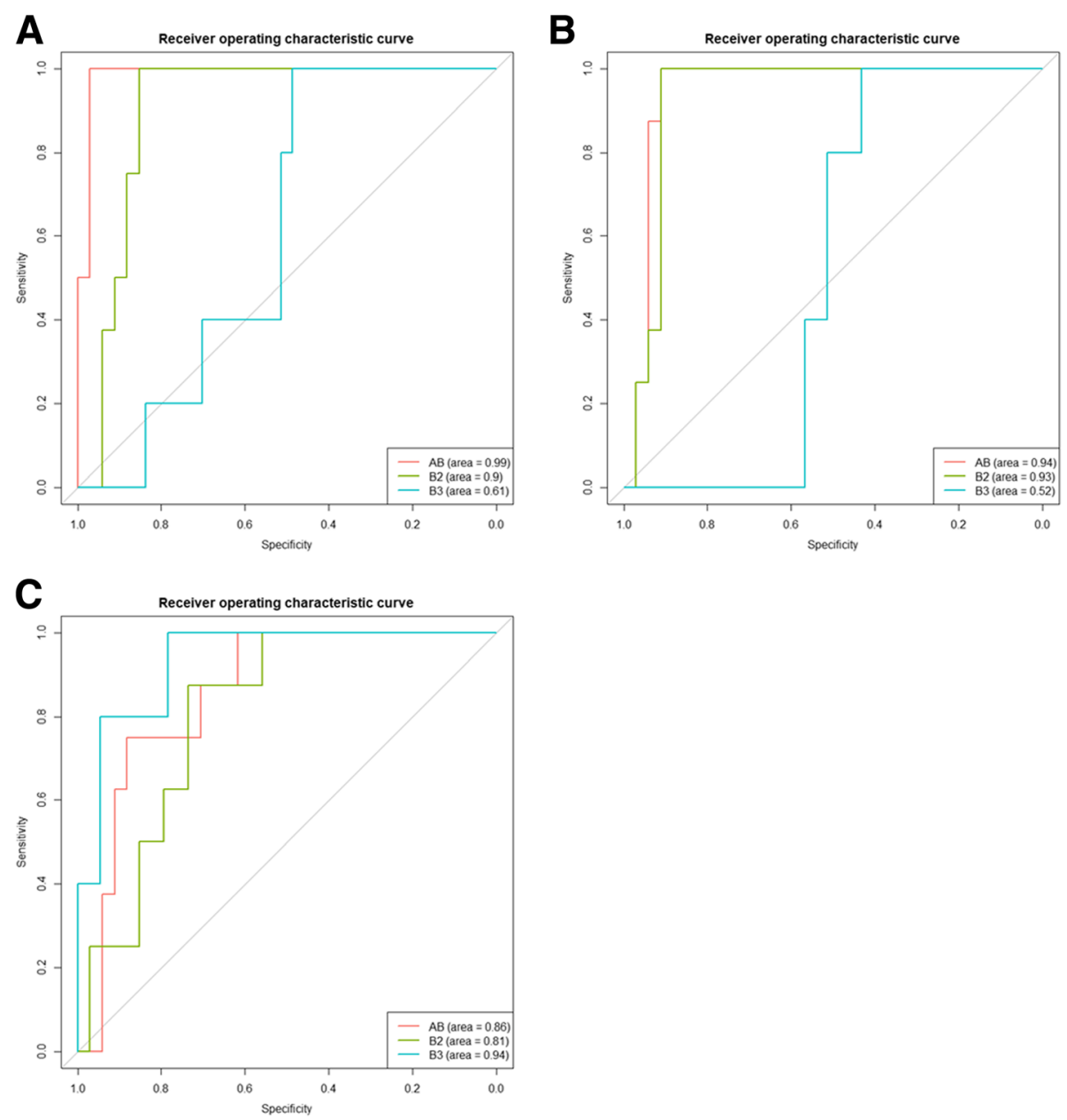

Fig. 2 Receiver operating characteristic curve of SVM classifiers to predict the type of thymoma. Differentially expressed proteins only in types $A B$ (a), B2 (b), and B3 (c) were served as test data to train different classifiers

subtype $\mathrm{AB}$ was also the best classifier among the 3 subtypes based on the ROC analysis.

In the beginning of the analysis, we excluded partial proteins $(<5 \%$ of total protein) as background noise. Although this may have slightly affected the results of the pathway analysis, small changes in these partial proteins often lead to tremendous fold changes and result in false positives. Moreover, although subtype AB, B2 and B3 are all mixed with various amount of lymphocytes, the contents of lymphocytes are not a concern because the classification is based on the differentially expressed proteins in each subtype. If the differentially expressed proteins come from lymphocytes, they are part of characteristics for classifying the subtypes of thymoma. Thirdly, although protein expression levels could change at different tumor stages, we did not consider stage as a factor in our analysis. The main reason for this was that the samples used for compiling the data set showed no significant deviation from randomness in the distribution across stages, and the main focus of this study was to classify subtypes of thymoma.
According to the "hallmarks of cancer" proposed by Hanahan and Weinberg in 2000, the molecular abnormalities of malignancies can be summed up in 6 characteristics: self-sufficiency in growth signaling; insensitivity to antigrowth signals; evading apoptosis; limitless replicative potential; sustained angiogenesis; and tissue invasion and metastasis [18]. In our study, pathway analysis revealed several canonical pathways related to signal transduction (6 of 15 pathways), nuclear receptor activation (4 of 15 pathways), and complement system (2 of 15 pathways) in the 3 thymoma types, which correlate with Hanahan and Weinberg's hallmarks of cancer.

The similarity of pathways between subtypes could be caused by similar genetic expression profiles between samples. As mentioned earlier, LXR/RXR activation and FXR/RXR activation were observed in common in subtypes B2 and B3, whereas acute phase response signaling and complement system were in common in subtypes $\mathrm{AB}$ and $\mathrm{B} 3$. These results correlate with the clustering analysis, where $A B$ was 


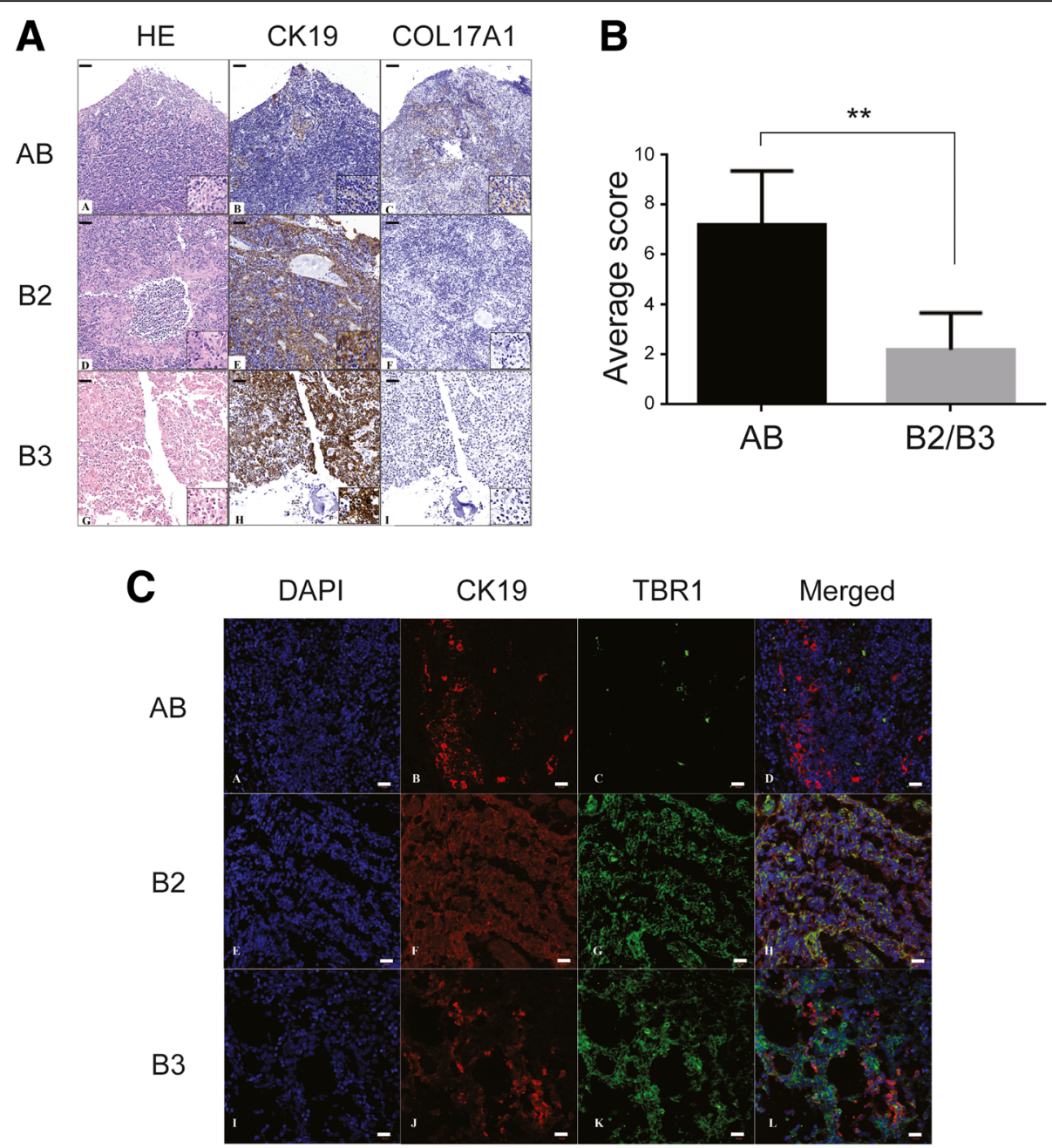

Fig. 3 Immunochemical analysis of COL17A1 and TBR1 in type AB and B2/B3 of thymoma. (a) immunohistochemistry of COL17A1. Formalin-fixed and paraffin-embedded (FFPE) tissue sections were stained to examine the presence of COL17A1. Scale bar: $100 \mu \mathrm{m}$. Insert magnification: 400X (b) Quantification of COL17A1. The immunoreactive score is determined by multiplication of the score of staining intensity with the score of percentage of positively stained cells. ${ }^{*}, P<0.01$. c Immunofluorescence of TBR1. CK19 (Cytokeratin 19): epithelial marker of thymoma. Scale bar: $20 \mu \mathrm{m}$

separate from the $\mathrm{B} 2+\mathrm{B} 3$ cluster, but closer to $\mathrm{B} 3$ than to B2.

The similarity between the top canonical pathways in subtype $A B$-acute phase response signaling, apoptosis signaling, and granzyme B signaling ( $-\log P$-value $=6.96$, 3.07 and 2.59)-suggests that the genetic expression profile changes in subtype $A B$ are highly related to signal transduction.

ROC analysis is useful for assessing the utility of predictors in clinical metrics and diagnostics tests. It is widely used in epidemiology and diagnostic radiology research [19-21]. So far this method has not yet been performed for thymic tumor classification. As far as we know, this is the first study applying ROC analysis to classify different types of thymoma. In our study, the AUC in all 3 tumor types was $\geq 0.93$, implying high performance of these classifiers. Despites of its high performance and being scrutinized by leave-one-out cross validation, if there are any other independent cohort available, it will still be a good reason to further improve its reliability without the use of original samples as statistical assessment. However, because a rare disease thymoma is, the difficulty of doing so is also higher than other common diseases.

In this study, we analyzed the protein mass spectrometry data of 21 Chinese patients with 3 types of thymoma (AB, B2 and B3), and developed a thymoma subtype predictive model with overall AUC $\geq 0.93$. Although there probably does not exist any geographic or racial difference in terms of histological classification of thymomas, the use of single human race may still be a limitation in this study. 
We expect to combine more clinical data (e.g., survival data) in the future to improve the predictive model. Since the current dilemma in classifying types of thymoma is the diverse clinical features, generation of a predictive model with good performance will be a considerable contribution to this field of study.

\section{Conclusions}

In this study, we identified protein signatures to predict the subtypes $\mathrm{AB}, \mathrm{B} 2 / \mathrm{B} 3$ of thymoma with high accuracy, sensitivity and specificity. One of the differentially expressed proteins COL17A1 was further validated using immunohistochemistry. These specific protein signatures could facilitate accurate diagnosis of thymoma patients.

\section{Abbreviations}

DEP: Differentially expressed protein; FFPE: Formalin-fixed and paraffinembedded; IPA: Ingenuity Pathway Analysis; LOOCV: Leave-one-out cross validation; ROC: Receiver operating characteristic; TCGA: The Cancer Genome Atlas; WHO: World Health Organization

\section{Acknowledgements}

Not applicable.

\section{Authors' contributions}

QLS, WF, and EYC conceived and designed the experiments. QLS and LZ performed the experiments. LCL, TPL, MHT, and YWH analyzed the data. WF and EYC contributed reagents, materials, and/or analysis tools. LCL, YAC, WF, and EYC wrote the paper. All authors read and approved the final manuscript.

\section{Ethics approval and consent to participate}

The study was approved by the Institutional Review Board of Shanghai Chest Hospital. And written consent was acquired from all patients included in this study.

\section{Funding}

This research was supported by a grant from the Ministry of Science and Technology (Grant No. MOST 102-2320-B-002-015, MOST 108-3017-F-002004-), Shanghai Municipal Natural Science Foundation (No.17ZR1426600), and Medical Engineering Cross Fund of Shanghai Jiao Tong University (No. YG2016MS80), Strategic Priority Research Program of the Chinese Academy of Sciences (No. XDA12020101), Interdisciplinary Program of Shanghai Jiao Tong University (No. ZH2018ZDA27), Shanghai Chest Hospital Project of Collaborative Innovation(YJXT20190104), Nurture projects for basic research of Shanghai Chest Hospital(2018YNJCM06). These funding sources had no role in the design of this study and will not have any role during its execution, analyses, interpretation of the data, or decision to submit results.

\section{Availability of data and materials}

All data generated or analyzed during this study are included in this published article.

\section{Consent for publication}

Not Applicable.

\section{Competing interests}

The authors declare that they have no competing interests.

\section{Author details}

${ }^{1}$ Graduate Institute of Physiology, College of Medicine, National Taiwan University, Taipei 10051, Taiwan. ${ }^{2}$ Department of Thoracic Surgery, Shanghai Chest Hospital, Shanghai Jiao Tong University, Shanghai 200030, China. ${ }^{3}$ Bioinformatics and Biostatistics Core, Center of Genomic and Precision Medicine, National Taiwan University, Taipei 10055, Taiwan. ${ }^{4}$ Department of Public Health, National Taiwan University, Taipei 10055, Taiwan. ${ }^{5}$ Institute of Biotechnology, National Taiwan University, Taipei 10672, Taiwan. ${ }^{6}$ Graduate
Institute of Biomedical Electronics and Bioinformatics, National Taiwan University, Taipei 10617, Taiwan. ${ }^{7}$ Department of Pathology, Shanghai Chest Hospital, Shanghai Jiao Tong University, Shanghai 200030, China.

${ }^{8}$ Department of Electrical Engineering, Graduate Institute of Biomedical

Electronics and Bioinformatics, National Taiwan University, Taipei 106, Taiwan.

Received: 25 January 2019 Accepted: 6 August 2019

Published online: 13 August 2019

\section{References}

1. MAd B, Roden AC, Marx A, Marino M. Histologic classification of Thymoma: a practical guide for routine cases. J Thorac Oncol. 2014;9:S125-30.

2. Rosai J, Sobin LH. Histological typing of tumours of the thymus. SpringerVerlag. 1999:65.

3. Strobel P, Marx A, Zettl A, Muller-Hermelink HK. Thymoma and thymic carcinoma: an update of the WHO classification 2004. Surg Today. 2005; 35(10):805-11.

4. Marx A, Strobel P, Zettl A, Chan J, Harris N, Kuo T: World Health Organization classification of tumours. Pathology and genetics of tumours of the lung, thymus and heart 2004, volumn 7:p.152-153.

5. Suster S, Moran CA. Thymoma, atypical thymoma, and thymic carcinoma. A novel conceptual approach to the classification of thymic epithelial neoplasms. Am J Clin Pathol. 1999;111(6):826-33.

6. Weis C-A, Yao X, Deng Y, Detterbeck FC, Marino M, Nicholson AG, Huang J, Ströbel P, Antonicelli A, Marx A. The impact of thymoma histotype on prognosis in a worldwide database. J Thorac Oncol. 2015;10(2):367-72.

7. Suster S, Moran CA. Thymoma ClassificationCurrent status and future trends. Am J Clin Pathol. 2006;125(4):542-54.

8. Kirchner T, Müller-Hermelink HK: New approaches to the diagnosis of Thymic epithelial tumors. In: Progress in surgical pathology: Volume X. edn. Edited by Cecilisa Fenoglio-Preiser M, Wolff M, Rilke F. Berlin, Heidelberg: Springer Berlin Heidelberg; 1989: 167-189.

9. Bernatz PE, Harrison EG, Clagett OT. Thymoma: a clinicopathologic study. J Thorac Cardiovasc Surg. 1961;42:424-44.

10. Radovich M, Pickering CR, Felau I, Ha G, Zhang H, Jo H, Hoadley KA, Anur P, Zhang J, McLellan M, et al. The integrated genomic landscape of Thymic epithelial tumors. Cancer Cell. 2018;33(2):244-58 e210.

11. Cancer Genome Atlas Research. N: Integrated genomic characterization of papillary thyroid carcinoma. Cell. 2014;159(3):676-90.

12. A genomics-based classification of human lung tumors. Sci Transl Med 2013, 5(209):209ra153.

13. Travis WD, Brambilla E, Burke AP, Marx A, Nicholson AG: Introduction to the 2015 World Health Organization classification of tumors of the lung, pleura, Thymus, and Heart Journal of thoracic oncology : official publication of the International Association for the Study of Lung Cancer 2015, 10(9):12401242.

14. Zettl A, Ströbel P, Wagner K, Katzenberger T, Ott G, Rosenwald A, Peters K, Krein A, Semik M, Müller-Hermelink H-K, et al. Recurrent genetic aberrations in Thymoma and Thymic carcinoma. Am J Pathol. 2000;157(1):257-66.

15. Lee GY, Yang WI, Jeung HC, Kim SC, Seo MY, Park CH, Chung HC, Rha SY. Genome-wide genetic aberrations of thymoma using CDNA microarray based comparative genomic hybridization. BMC Genomics. 2007;8:305.

16. Comprehensive molecular profiling of lung adenocarcinoma. Nature 2014, 511(7511):543-550.

17. Masaoka A, Monden Y, Nakahara K, Tanioka T. Follow-up study of thymomas with special reference to their clinical stages. Cancer. 1981;48(11):2485-92.

18. Hanahan D, Weinberg RA. The hallmarks of Cancer. Cell. 2000;100(1):57-70.

19. Greiner M, Pfeiffer D, Smith RD. Principles and practical application of the receiver-operating characteristic analysis for diagnostic tests. Preventive Veterinary Medicine. 2000;45(1):23-41.

20. Lind PA, Marks LB, Hollis D, Fan M, Zhou S-M, Munley MT, Shafman TD, Jaszczak RJ, Coleman RE. Receiver operating characteristic curves to assess predictors of radiation-induced symptomatic lung injury. Int J Radiat Oncol Biol Phys. 2002;54(2):340-7.

21. Metz CE. Basic principles of ROC analysis. Semin Nucl Med. 1978;8(4):283-98.

\section{Publisher's Note}

Springer Nature remains neutral with regard to jurisdictional claims in published maps and institutional affiliations. 